\title{
Data intelligence is the future of monitoring
}

\author{
Andrew J. E. Seely
}

Published online: 5 February 2014

(C) Springer Science+Business Media New York 2014

Uncovering otherwise hidden clinically valuable knowledge from routinely monitored and commonly discarded data is the objective of intelligent biosignal analysis. In any Intensive Care Unit (ICU), a bedside monitor routinely provides a continuous display of waveforms and point-intime display of vital signs. Typically, hundreds of data points are recorded per second (125 per waveform), however care providers typically chart hourly and detect changes over several hours. The inefficient waste is remarkable, that is, if one believes there is valuable information hidden within the waveform. Addressing this challenge, there is a growing and exciting science driven by the desire to transform the way waveforms are analyzed, improve the value of monitoring, and thus improve quality and efficiency of care.

In interpreting this editorial regarding the accompanying article by Lake and colleagues [1], it is important to disclose my bias and conflict of interest regarding this work and subject matter. I have been long pursuing the development of software to perform multi organ variability monitoring and testing it clinically, seeking to understand the physiology of altered variability, and pursuing the commercial objective of introducing variability-based products to ICUs. Thus, I am biased based on time invested, potential for financial benefit, and my belief system; I do this work because of underlying beliefs that this

This Editorial refers to the article available at doi: $10.1007 / \mathrm{s} 10877-$ 013-9530-x.

\footnotetext{
A. J. E. Seely $(\bowtie)$

Divisions of Thoracic Surgery and Critical Care Medicine, Ottawa Hospital Research Institute, University of Ottawa, Ottawa Hospital, General Campus, 501 Smyth Road, Box 708, Ottawa, ON K1H 8L6, Canada e-mail: aseely@ohri.ca
}

approach could help provide clinically meaningful information to the bedside to improve care for our patients. Thus, please interpret these comments as you see fit.

To uncover clinically meaningful information from waveforms (e.g. electrocardiogram, capnography), two steps are required. The first is waveform analytics. The anatomy of a waveform must be dissected and measured per beat or breath. Data derived from waveform anatomy per breath such as ST-depression and end-tidal $\mathrm{CO} 2$ monitoring are routinely available and commonly clinically used. A second level of analysis involves the integration of information over intervals-of-time, with analyses degree and character of variation of the time series of events captured by the waveform (e.g. inter-beat and inter-breath intervals). Variability analyses include measures of overall variation, patterns of accelerations and decelerations, presence of high and low frequency variation, scale-free correlations, and more. All of these measures are nonetheless derived from analyzing waveforms. To transform these measures into clinically useful information, one needs a fundamentally distinct second step, namely populationderived predictive modeling. In contrast to waveform analytics, predictive modeling requires the time consuming and expensive process of enrolling patients into large studies, recording outcomes, as well as measuring the waveform analytics in a reproducible, standardized fashion. Thus, the combination of biomedical engineering and statistical modeling are employed in the act of uncovering clinical decision support from waveform biosignals to and from the bedside.

Guiding the way in this domain of bedside delivery of complex signal bioinformatics is a group from the University of Virginia, led by Randall Moorman and Douglas Lake. They sought to improve neonatal critical care monitoring by developing automated methods to uncover 
decelerations and decreased variation of heart rate timeseries in neonates developing sepsis. They developed measures of variation optimally tailored to this unique patient population, derived a predictive model called the HeRO score (which provides a measure of risk of subsequent deterioration in the next $24 \mathrm{~h}$ ), validated it in an independent cohort, and evaluated its impact in a large, multi-center randomized controlled trial. Moorman and Lake and all of their colleagues that contributed should be heartily congratulated for the remarkable achievement of the derivation of the HeRO score in 1,022 patients in two centers, validation in 1,489 patients in nine ICUs, and most importantly completion of a 3,003 patient multicenter randomized controlled trial, also in nine ICUs. This herculean journey began over 15 years ago, and is a testament to the fact that passion drives innovative research.

What does this article provide in new information? The article by Lake et al. [1] provides a more in depth statistical analysis of the following domains: (1) they provide the measure of risk provided by the novel marker model, namely the HeRO score (along with $95 \% \mathrm{CI}$ and $p$ values), after adjustment for known risk factors (along with $95 \% \mathrm{CI}$ and $p$ values), and the $p$ value difference in comparison to a standard marker model; (2) they analyze the discrimination of the marker, using several accepted statistical methods to do so with or without the added marker; and (3) evaluate the accuracy of the marker, comparing observed vs. expected event rates) and reporting the number of reclassified subjects. This is in keeping with the recommendations of Hlatky et al. [2] whom developed a comprehensive framework to evaluate the added value of a biomarker, on behalf of the American Heart Association. Indeed, the principal contribution of the accompanying manuscript by Lake, Fairchild and Moorman [1] is that they explicitly provide an analysis of their novel biomarker in accordance with the methods developed by Hlatky et al. [2].

How does one uncover and fully evaluate a novel biomarker or in this case a monitoring technology? In addition to laying out the methods for biostatisitcal evaluation, Hlatky et al. also highlight the stages of development of a research program designed to comprehensively evaluate the added value of a novel biomarker technology. These stages include: (1) Proof of concept-Do novel marker levels differ between subjects with and without outcome?

(2) Prospective validation-Does the novel marker predict development of future outcomes in a prospective cohort or nested case-cohort/case-cohort study? (3) Incremental value-Does the novel marker add predictive information to established, standard risk markers? (4) Clinical utilityDoes the novel risk marker change predicted risk sufficiently to change recommended therapy? (5) Clinical outcomes-Does use of the novel risk marker improve clinical outcomes, especially when tested in a randomized clinical trial? (6) Cost-effectiveness-Does use of the marker improve clinical outcomes sufficiently to justify the additional costs of testing and treatment? [2].

The last two stages require a randomized controlled trial. While unequivocally necessary, the optimal design of a trial to investigate a novel monitoring technology remains controversial. Both protocolled and pragmatic approaches have merit and limitations. A protocolized randomized controlled trial, for example as exhibited by Rivers et al. [3] evaluates the impact of a protocol (e.g. goal-directed based on a monitored value) to guide interventions, introduced simultaneously alongside a monitor. In contrast, a pragmatic randomized controlled trial, where patients are exposed simply to the presence or absence of monitoring, provides an attempt to assess the 'real world' impact of a novel monitor at the bedside in isolation. As detailed by Lake et al. [1], the HeRO monitor was introduced at the bedside in a randomized fashion with no mandated response to the values being displayed [4]. As no intervention is mandated by its results, it is subject to variable interpretation and variable interventions by care providers. The value of the pragmatic trial is that it allows physicians to integrate the monitoring information with their clinical, laboratory and radiologic gestalt in a dynamic and individualized manner to make decisions accordingly. The limitation is that ignoring the monitor or erroneous interpretations will dampen the observed signal of potential benefit. Both trial designs are subject to the confounding impact of increased attention and/or care as a result of the monitoring. While many factors guide the optimal choice for a protocolled or pragmatic trial, the potential impact on knowledge translation should be taken into consideration, as adoption is the ultimate objective of clinical research of novel monitoring technology.

From a general point of view, what level of evidence is necessary to drive adoption? In general, evidence from a single RCT is enough to change practice in a large number of adopters, in particular if it is large and multicenter. In general, further clinical trials are recommended to ascertain the initially demonstrated value. Although multiple randomized controlled trials provide far greater proof of value than a single clinical trial, they are time consuming, costly and may not offer new information, and thus provide less value for the investment. Relevant to this discussion, perhaps an argument for a second clinical trial of the HeRO score would be to evaluate a protocolled approach to responding to HeRO monitoring.

Regardless of evidence, there are barriers to adoption that can reduce or stop adoption. Selective gut decontamination evidently has barriers to adoption that trump the compelling evidence. Numerous barriers and accelerants exist in knowledge translation and adoption of novel technology. Existing opinion (i.e. our priors) regarding the 
technology and its physiologic meaning are critically important to individual acceptance. All evidence is interpreted through the lens of our perceptions, beliefs and biases, and then standards of care are created via collective interpretation and prioritization. Second, there is the fundamental requirement that novel monitoring technologies integrate within processes of care in an accessible manner. Innumerable other factors that impact adoption exist, however there is one of particular importance to innovations regarding the process of care delivery, such as monitoring.

Commonly, experiential, human-interaction and individual patient driven change processes are essential to assist adoption. Atul Gwande highlights these lessons in a recent New Yorker article entitled "Slow Ideas" [5]. Physical interaction, "boots on the ground", participation in care and mentoring are enormously helpful to educate and guide care providers with the use of a novel technology. Through these interactions, care providers need to experience the improvement of care of individual patients offered by a biomarker or monitoring technology. The monitor needs to tell the clinician something helpful that they did not already know, and that it helped in the care of an individual patient. The personal experience of having improved the patient's care through knowledge derived from the monitor is personal evidence of its benefit. While pundits of evidencebased-medicine may claim this experience is not true evidence; regardless, it is my impression that it is acted upon as if it were evidence. Our experience certainly affects our priors, which affects the means we interpret population evidence. When personal and population evidence harmoniously collide, such as with early goal directed therapy for septic shock, then adoption is rapid and widespread. Not reported in the adjoining article is the experience of clinicians using the HeRO score for neonatal monitoring, positive and negative. That experience would be worthy of a separate and quite different publication, which might further augment the adoption of this technology.

\section{References}

1. Lake DE, Fairchild KD, Moorman JR. Evaluation of heart rate characteristics monitoring as a novel risk marker for neonatal sepsis. J Clin Monit Comput. 2014.

2. Hlatky MA, Greenland P, Arnett DK, et al. Criteria for evaluation of novel markers of cardiovascular risk: a scientific statement from the American Heart Association. Circulation. 2009;119(17):2408-16.

3. Rivers E, Nguyen B, Havstad S, et al. Early goal-directed therapy in the treatment of severe sepsis and septic shock. N Engl J Med. 2001;345(19):1368-77.

4. Moorman JR, Carlo WA, Kattwinkel J, et al. Mortality reduction by heart rate characteristic monitoring in very low birth weight neonates: a randomized trial. J Pediatr. 2011;159(6):900-6.

5. Gawande A. Slow Ideas. Some Innovations spread fast. How do you speed the ones that don't? The New Yorker. New York, 2013. 